\title{
Metabolic comorbidities of psoriasis (Review)
}

\author{
DACIANA ELENA BRANISTEANU ${ }^{1 *}$, RUXANDRA ANGELA PIRVULESCU $^{2,3}$, ALINA ELENA SPINU $^{4}$, \\ ELENA ANDRESE PORUMB ${ }^{5}$, MIHAELA COJOCARU $^{4}$, ALIN CODRUT NICOLESCU $^{6 *}$, \\ DANIEL CONSTANTIN BRANISTEANU ${ }^{7}$, CATALINA IOANA BRANISTEANU ${ }^{8 *}$, \\ ANDREEA DIMITRIU $^{9}$, ANISIA IULIANA ALEXA ${ }^{10}$ and MIHAELA PAULA TOADER ${ }^{11}$ \\ ${ }^{1}$ Department of Dermatology, 'Grigore T. Popa’ University of Medicine and Pharmacy, 700115 Iasi; \\ ${ }^{2}$ Department of Ophthalmology, 'Carol Davila' University of Medicine and Pharmacy, 050474 Bucharest; \\ ${ }^{3}$ Ophthalmology Clinic, Emergency University Hospital, 050098 Bucharest; \\ ${ }^{4}$ Department of Dermatology, Railway Clinical Hospital, 700506 Iasi; ${ }^{5}$ Department of Dermatology, \\ 'Sf. Spiridon' Clinical Emergency County Hospital, 700111 Iasi; ${ }^{6}$ Department of Dermatology, \\ 'Roma' Medical Center for Diagnosis and Treatment, 011773 Bucharest; ${ }^{7}$ Department of Ophthalmology and \\ ${ }^{8}$ Faculty of Medicine, 'Grigore T. Popa' University of Medicine and Pharmacy, 700115 Iasi; \\ ${ }^{9}$ Department of Dermatology, 'Arcadia' Hospitals and Medical Centers, 700620 Iasi; \\ Departments of ${ }^{10}$ Ophthalmology and ${ }^{11}$ Oral Dermatology, \\ 'Grigore T. Popa' University of Medicine and Pharmacy, 700115 Iasi, Romania
}

Received October 15, 2021; Accepted November 16, 2021

DOI: $10.3892 / \mathrm{etm} .2021 .11102$

\begin{abstract}
Psoriasis is a chronic inflammatory and immune-mediated condition, which is no longer considered as being limited to the skin, but may affect the entire body. Epidemiological studies have shown that certain disorders, including obesity, diabetes, liver abnormalities, elevated lipid levels in the blood and metabolic syndrome, may occur more frequently in patients with psoriasis compared with the general population. As psoriasis is a chronic disease, the frequently associated comorbidities must be identified early to ensure timely treatment and, possibly, their prevention. Comorbidities often manifest clinically 1-2 years after the onset of psoriasis and are commonly seen in patients with severe forms of the disease. The association between psoriasis and its comorbidities is not coincidental, but rather based on common pathophysiological mechanisms and risk factors that
\end{abstract}

Correspondence to: Dr Alina Elena Spinu, Department of Dermatology, Railway Clinical Hospital, 1 Garabet Ibraileanu Street, 700506 Iasi, Romania

E-mail: alinaelenapalie@yahoo.com

Dr Ruxandra Angela Pirvulescu, Department of Ophthalmology, 'Carol Davila' University of Medicine and Pharmacy, 37 Dionisie Lupu Street, Sector 2, 050474 Bucharest, Romania

E-mail: ruxandra_pascu@yahoo.co.uk

*Contributed equally

Key words: psoriasis, metabolic comorbidities, management, obesity, diabetes mellitus, dyslipidemia, metabolic syndrome underlie the increased frequency of comorbidities in patients with psoriasis. The aim of the present review was to emphasize the important role of dermatologists in the early recognition of comorbidities in patients with psoriasis, with a focus on metabolic comorbidities, precisely because the dermatologists are usually the first medical contact due to the predominance of skin lesions. Therefore, these specialists have the responsibility to inform patients on the association between psoriasis and possible multiple comorbidities, devise prevention and treatment plans, or even redirect patients to other specialists.

\section{Contents}

1. Introduction

2. Metabolic comorbidities of psoriasis

3. Management of metabolic comorbidities in psoriasis

4. Conclusions

\section{Introduction}

Psoriasis is a chronic immune-mediated inflammatory disease, characterized by keratinocyte proliferation, that is characterized by well-defined red plaques with silvery-white scales, which can involve any region of the skin (and other components of the integumentary system, including the nails), but is usually located on the elbows, knees, scalp and presacral region $(1,2)$. With a prevalence of $0.1-3 \%$ in various populations, psoriasis can start at any age (but often presents between 15 and 30 years) and appears to be equally common in both male and female patients $(1,2)$. As regards the pathogenesis of psoriasis, it involves a genetic component (association with 
HLA-Cw6 and a positive family history) and an immune dysfunction, with the added contribution of numerous environmental factors (including infections, trauma, medications and psychological stress) (3). The immune response is characterized by the proliferation of T helper (Th)1, Th17 and Th22 cells, which results in the production of proinflammatory mediators, including IFN- $\gamma$, IL-6, IL-22 and TNF- $\alpha$, which serve an important role in maintaining chronic inflammation in psoriasis $(2,4,5)$.

Although skin involvement is often the most prominent manifestation, the recognition of psoriasis as a multisystemic chronic inflammatory disorder is essential for early identification of comorbidities. Moderate to severe psoriasis is frequently associated with a higher incidence of serious comorbidities, including psoriatic arthritis, cardiovascular diseases, metabolic disorders, malignancy and severely compromised quality of life $(1,6,7)$. The association of metabolic comorbidities, or even metabolic syndrome, with psoriasis has been suggested, as psoriatic patients, particularly women, have at least twice the risk of developing these comorbidities compared with the general population. Among the metabolic comorbidities, those most commonly associated with psoriasis are obesity, diabetes, non-alcoholic fatty liver disease, atherogenic dyslipidemia and metabolic syndrome, the latter comprising a constellation of disorders that include abdominal obesity, high blood pressure, dyslipidemia and increased insulin resistance, which are all important cardiovascular risk factors (8).

A literature search was conducted using the electronic databases Google Scholar, PubMed, Key Elsevier, UpToDate and Medscape for the terms 'psoriasis' in combination with 'metabolic comorbidities', 'management of psoriasis', 'obesity', 'diabetes mellitus', 'metabolic syndrome' and 'dyslipidemia'. A total of 54 publications found in the literature, including case reports, case series and literature review-type papers, were herein combined into a concise report of psoriasis-related metabolic comorbidities.

\section{Metabolic comorbidities of psoriasis}

Obesity is a complex chronic disease that affects a large proportion of the general population. This condition is attributed to both genetic and environmental factors, and is measured by the body mass index (BMI=weight in $\mathrm{kg}$ divided by the square of the height in $\mathrm{m}$ ), with higher BMI values correlating with a higher percentage of body fat (9). The most relevant parameters for obesity are the degree of intra-abdominal fat (visceral adiposity) and increased waist circumference, which independently constitute an increased risk of type 2 diabetes mellitus, cardiovascular disease, hypertension, sleep apnea, stroke and certain types of cancer, with reduced life expectancy $(10,11)$. Obesity is one of the comorbidities commonly associated with psoriasis; it is more prevalent among patients with severe compared with mild psoriasis, and approximately twice as common among patients with psoriasis compared with the general population, with numerous studies showing that patients with psoriasis are more frequently overweight (BMI $\geq 25$ but $<30 \mathrm{~kg} / \mathrm{m}^{2}$ ) or even obese $\left(\mathrm{BMI} \geq 30 \mathrm{~kg} / \mathrm{m}^{2}\right)(3,6,12-14)$. Obesity is recognized as an independent risk factor for psoriasis, but is also an important risk factor for cardiovascular disease, type 2 diabetes mellitus, obstructive sleep apnea and osteoarthritis, and it is a leading cause of premature death, with recent population-based studies showing an increasing number of adults and children affected (12,15-19). It has long remained unclear whether obesity is a risk factor for the development of psoriasis or its consequence. However, regardless of cause and effect, it has been found that there is an association between obesity and the development and progression of psoriasis (increased BMI is associated with a higher degree of psoriasis severity) $(2,20-22)$. Given the fact that the prevalence of psoriasis is increasing while the genetic basis has remained the same, it is believed that environmental factors, including lifestyle and dietary habits, which are modifiable risk factors in the pathogenesis of psoriasis, play an important role in this growing prevalence $(2,22)$. In industrialized countries, high-fat, high-salt and high-sugar diets with excessive caloric intake are increasingly promoted, resulting in food intake that is inversely proportional to energy consumption, ultimately leading to the storage of excess energy deposits as body fat (2).

Regarding the mechanisms underlying the obesity-psoriasis association, they comprise a common chronic inflammatory condition, low physical activity, poor eating habits and high alcohol consumption, possibly resulting from the psychosocial impact of psoriasis on patients. Increased release of free fatty acids from white adipocytes, which, in turn, lead to increased serum fatty acid levels, is a key component in the pathogenesis of several diseases associated with obesity, including psoriasis. Adipose tissue is considered to be a true endocrine organ that secretes a multitude of soluble mediators involved in immunity, inflammation, and metabolic and appetite regulation $(2,22)$. Adipokines (mediators secreted by adipose tissue) include classic cytokines, such as IL-6, TNF- $\alpha$ and leptin-specific molecules, adiponectin, resistin and visfatin, which exert proinflammatory effects that may contribute to the inflammatory status in psoriasis, which may explain the increased risk of psoriasis in obese patients $(2,20,22,23)$. Thus, weight loss in these patients may reduce the risk and severity of psoriasis by lowering the levels of leptin and proinflammatory cytokines $(20,23)$. Another feature that supports the coexistence of obesity with psoriasis is the presence of the HLA-Cw6 gene; this is a major psoriasis sensitivity gene, which is also associated with obesity and has been shown to increase the risk of patients developing psoriasis (2).

As regards treatment, studies have shown the association of obesity with a low response to systemic and biological treatment for psoriasis, but also with numerous side effects when obesity is associated with other conditions (such as metabolic syndrome and non-alcoholic steatohepatitis) $(2,9,24)$. The hepatotoxic side effects of methotrexate therapy are more aggravated by the association with obesity rather than by the association with viral hepatitis or alcohol consumption, which necessitates rigorous monitoring of hepatic transaminase levels. At the same time, obesity in the long term can lead to the loss of the therapeutic response to methotrexate (2). During systemic therapy with cyclosporine (a lipophilic drug), obesity was shown to exacerbate nephrotoxicity due to elevated serum levels of cyclosporine in overweight or obese patients (21). Biological anti-TNF- $\alpha$ agents, which currently form the basis of psoriasis therapy, are also affected by increased BMI. Some studies have shown that the decrease in the psoriasis area and severity index (PASI) during therapy with anti-TNF- $\alpha$ agents 
has been negatively affected in patients with elevated BMI; on the other hand, this therapy may lead to weight gain of treated patients, possibly due to the reduced release of leptin, which causes hyperphagia $(2,24)$.

Diabetes mellitus is a group of metabolic disorders characterized by hyperglycemia resulting from insufficient secretion or decreased action of insulin (25). The diagnostic criteria for diabetes are as follows: Symptoms of diabetes associated with occasional plasma glucose concentration $\geq 200 \mathrm{mg} / \mathrm{dl}$ or elevated fasting plasma glucose level $\geq 126 \mathrm{mg} / \mathrm{dl}$ or 2 -h postload glucose $\geq 200 \mathrm{mg} / \mathrm{dl}$ (25). Glycated hemoglobin (HbA1c) level is another measure used to reflect the average plasma glucose levels over the previous 6-10 weeks $(15,25,26)$. An HbAlc level $\geq 6.5 \%$ ( $\geq 48 \mathrm{mmol} / \mathrm{mol}$ ) is considered sufficient for diagnosing diabetes mellitus (26). As diabetes itself is a cardiovascular risk factor, when associated with psoriasis it carries a significant risk of metabolic syndrome or cardiovascular events $(16,25,26)$. Several studies have evaluated and reported the association between psoriasis and diabetes, suggesting a probable pathophysiological link between the two diseases $(22,23,27)$. The increase in the levels of TNF- $\alpha$, a proinflammatory cytokine that plays an important role in the pathogenesis of insulin resistance by reducing the tyrosine kinase activity of the insulin receptor, is a pathogenetic link between psoriasis and type 2 diabetes (23). Furthermore, in some studies, the upward regulation of the IL-23/Th17 axis appears to be key to the explanation of the cardiometabolic comorbidities in psoriasis $(17,21,23)$. Certain altered immune pathways in psoriasis, such as T-cell subtype 1 pathways and irregular oxidative and angiogenic mechanisms, may also predispose to reduced glucose tolerance and diabetes (27). Although it is unclear whether diabetes precedes psoriasis, most studies on the incidence of diabetes mellitus suggest that the majority of the patients develop diabetes after a diagnosis of psoriasis, with the highest risk occurring in patients with a severe form of psoriasis, who appear to be more likely to develop microand macrovascular complications compared with patients without psoriasis $(11,27)$.

Studies have shown that psoriasis and type 2 diabetes share common risk factors, and individuals who develop psoriasis at a younger age have a significantly increased risk of type 2 diabetes, which further emphasizes that psoriasis is a systemic inflammatory disorder $(13,15,27)$. As psoriasis is a chronic inflammatory disease associated with the expansion and activation of Th1 and Th17 T cells in the skin, with the production of cytokines such as TNF- $\alpha, \mathrm{C}$-reactive protein (CRP) and IL-6, an inflammatory basis for diabetes has also been identified and low-grade inflammation has been shown to precede and predict the development of insulin resistance and diabetes. Thus, inflammatory cytokines, such as IL- 6 and TNF- $\alpha$, have been associated with insulin resistance and the onset of type 2 diabetes $(5,13)$. Moreover, adipocytokines (leptin and adiponectin), obesity (increased BMI), as well as behavioral characteristics, such as smoking, play key roles in the association of psoriasis with diabetes $(13,20,28)$. In summary, the immune-mediated inflammatory process, metabolic biomarkers and environmental factors are the main potential factors underlying the association between psoriasis and diabetes. Patients with psoriasis, particularly those with a severe form, should be educated regarding the increased risk of developing diabetes, and dermatologists should recommend more intensive screening in order to identify the onset of diabetes and administer treatment in a timely manner (27).

Patients with psoriasis have a markedly higher risk of developing liver abnormalities compared with the general population, the most common being non-alcoholic fatty liver disease (NAFLD), alcoholic hepatitis, drug-induced hepatitis and neutrophilic cholangitis (29). The manifestations of NAFLD may range from hepatic steatosis, which has a favorable prognosis, to non-alcoholic steatohepatitis, which may result in fibrosis, cirrhosis and hepatocellular carcinoma over time. Previous studies revealed that the prevalence of ultrasound-diagnosed NAFLD was notably higher among patients with psoriasis, regardless of daily alcohol consumption, smoking history, serum levels of alanine aminotransferase or the presence of metabolic syndrome $(22,30)$. It has been reported in the literature that patients with psoriasis develop more severe forms of NAFLD (steatohepatitis and advanced fibrosis) and, at the same time, patients with NAFLD tend to suffer from more severe forms of psoriasis. The severe forms of NAFLD are more commonly associated with exacerbated systemic/hepatic insulin resistance, atherogenic dyslipidemia and the release of proinflammatory, procoagulant, pro-oxidant and profibrogenic mediators [CRP, fibrinogen, IL-6, plasminogen activator inhibitor-1 (PAI-1) and TGF- $\beta$ ], which negatively affect the severity of psoriasis through increased proliferation of keratinocytes, maintenance of inflammation and upregulation of various vascular adhesion molecules (30). All these changes represent the true pathogenic links between NAFLD and psoriasis, which are a common finding in diseases associated with psoriasis $(22,30)$.

Liver comorbidities limit treatment options for psoriasis; therefore, they require early identification and continuous monitoring. As NAFLD has a fairly high prevalence among patients with psoriasis, potentially hepatotoxic drugs (e.g., methotrexate and acitretin) should be administered with caution to patients with these diseases. NAFLD is a relative contraindication to methotrexate treatment, but a more aggressive monitoring of liver function can be achieved by liver biopsies at the time of initiation and at a cumulative dose of 1.0-1.5 g methotrexate (10). Non-invasive tests can also be used to detect liver fibrosis, including serological tests (Fibrotest and Fibroscan) and radiological imaging (ultrasound-based elastography, magnetic resonance elastography, pulsed acoustic radiation imaging and cross-sectional imaging), but without guaranteeing the safety of long-term methotrexate therapy $(10,29)$.

In conclusion, dermatologists should be aware not only of the high prevalence of NAFLD in patients with severe psoriasis, but also that liver function can be affected in psoriasis, both due to the disease itself and through several other associated factors $(18,25)$. The presence of other comorbidities is also a risk factor for impaired liver function (diabetes mellitus due to insulin resistance, which is an etiological factor for NAFLD, obesity and signs of metabolic syndrome, among others); in addition, hepatotoxic medication for the treatment of psoriasis and other comorbidities may cause impaired hepatic function $(22,29)$. 
Atherogenic dyslipidemia, characterized by elevated plasma concentrations of triglycerides, very low-density lipoproteins and low-density lipoproteins (LDL), as well as low serum concentrations of high-density lipoprotein (HDL) cholesterol, has been reported by numerous studies in association with psoriasis, particularly severe forms of the disease (26,31-33). These altered plasma lipid concentrations were frequently observed at the onset of psoriasis, being independent of sex, age, BMI, blood pressure, smoking or alcohol consumption, which may suggest that dyslipoproteinemia may be genetically determined, with apolipoprotein E gene polymorphism already being reported in association with the hyperlipidemia observed in psoriasis $(32,33)$. HDL-cholesterol, which is considered as the 'good cholesterol', is responsible for reverse cholesterol transport and has antioxidant and anti-inflammatory properties through regulating dendritic cell differentiation and reducing T-cell activation and IL-12 production (22). However, under conditions of chronic inflammation, such as in psoriasis, these beneficial effects are greatly reduced, but may be restored by psoriasis therapy (22). On the other hand, LDL-cholesterol, referred to as the 'bad cholesterol', accumulates in the blood vessels, where it generates reactive oxygen species and affects the function of vascular endothelial cells; in addition, its increase is associated with the increase of TNF- $\alpha$ levels in severe forms of psoriasis $(22,34)$. However, the detection of an abnormal lipid profile in psoriasis may also be attributed to anti-psoriasis therapy (retinoids or cyclosporine) or impaired digestive function (increased lipid absorption) (33). Abnormal lipid metabolism has been considered as an important etiopathogenic factor in psoriasis. The production of proinflammatory cytokines that underlie the pathophysiology of psoriasis also contribute to obesity, insulin resistance, dyslipidemia, endothelial dysfunction and increased oxidative stress, thereby exerting pro-atherogenic effects $(32,33)$.

One of the most significant comorbidities found in patients with psoriasis is the metabolic syndrome, a pathological condition with a wide range of manifestations, such as visceral obesity, insulin resistance/elevated fasting glucose level, dyslipidemia (elevated LDL-cholesterol, decreased HDL-cholesterol and elevated triglyceride levels) and hypertension, which represent major risk factors for cardiovascular diseases (8,35-37). There are some differences between the major criteria for the clinical diagnosis of metabolic syndrome, but the latest guidelines (IDF/NHBLI/AHA/World Heart Federation/International Atherosclerosis Society/International Association for the Study of Obesity, 2009) propose the presence of $\geq 3$ of the following 5 criteria: Increased waist circumference with population-specific and country-specific criteria (in Europid $>94 \mathrm{~cm}$ for men or $>80 \mathrm{~cm}$ for women), triglyceride level $\geq 1.7 \mathrm{mmol} / 1(\geq 150 \mathrm{mg} / \mathrm{dl})$ or drug treatment, HDL cholesterol $<1.0 \mathrm{mmol} / 1(<40 \mathrm{mg} / \mathrm{dl})$ in men or $<1.3 \mathrm{mmol} / \mathrm{l}(<50 \mathrm{mg} / \mathrm{dl})$ in women or drug treatment, high blood pressure $\geq 130 / 85 \mathrm{mmHg}$ or drug treatment and fasting plasma glucose $\geq 5.6 \mathrm{mmol} / 1(\geq 100 \mathrm{mg} / \mathrm{dl})$ or drug treatment $(8,19,22,38)$. Metabolic syndrome can be associated with moderate-to-severe psoriasis with a high prevalence $(\sim 50 \%)$, with patients with psoriasis (women more than men) having at least twice the risk of associating metabolic syndrome or individual components of the metabolic syndrome compared with patients without psoriasis $(8,9,12,18,38)$. It remains unclear whether the metabolic syndrome and its components are the cause or consequence of psoriasis, as lifestyle changes that may contribute to the occurrence of metabolic disorders can be adopted before or after the onset of psoriasis, hence the uncertainty in terms of temporal association (18). Furthermore, considering the psychosocial impact of psoriasis on patients (such as emotional disorders, anxiety and depression), changing harmful lifestyle choices may prove challenging.

The association between psoriasis and metabolic syndrome is not merely a coincidence. The identification of the pathophysiological mechanisms underlying these two diseases is of clinical importance, as it can uncover new therapeutic approaches (37-39). The factors that explain the association of the two diseases are a combination of the genetic component, the common immune-inflammatory pathways, environmental factors, smoking, alcohol consumption, psychological stress and low physical activity $(8,18,38,40,41)$. Systemic inflammation occurs both in psoriasis and metabolic syndrome, with inflammatory markers being elevated in both diseases. As mentioned, psoriasis is a chronic inflammatory disease, mediated by $\mathrm{T}$ cells and characterized by the expansion and activation of Th-1, Th-17 and Th-22 cell subpopulations, which causes the production of proinflammatory mediators (such as TNF- $\alpha$, IL-6, IL-1, IL-17, IL-22, IL-23, VEGF and IFN- $\gamma$ ) by lymphocytes and keratinocytes in the skin (40). These proinflammatory mediators are produced locally, but they can migrate into the systemic circulation, causing insulin resistance, circulatory endothelial dysfunction, increased oxidative stress, increased angiogenesis and hypercoagulation, which are processes that underlie inflammatory conditions, such as cardiometabolic comorbidities $(40,42)$. The most representative risk factors for the onset of metabolic syndrome are abdominal obesity (a major source of several proinflammatory cytokines and adipokines) and insulin resistance (significantly higher risk in psoriasis) (40). Although the literature supports an association between psoriasis and insulin resistance/type 2 diabetes, it should not be overlooked that obesity, particularly abdominal obesity, is closely associated with the development of metabolic syndrome and type 2 diabetes (43). Adipocytes are stimulated to release non-esterified fatty acids (NEFAs) by activated macrophages and $\mathrm{T}$ cells that reach the abdominal visceral adipose tissue (40). The continuous release of high levels of proinflammatory and/or prothrombotic factors from the abdominal visceral adipose tissue, such as IL-6, TNF- $\alpha$, leptin, resistin, chemerin, PAI-1, fibrinogen and CRP, along with the low levels of anti-inflammatory factors, such as adiponectin, contribute to the development of systemic insulin resistance, dysglycemia, atherogenic dyslipidemia, vascular dysfunction and NAFLD $(40,42,43)$. This proinflammatory/prothrombotic status has also been found in psoriasis, particularly in patients with increased disease activity, although some studies show elevated levels of proinflammatory markers even during remission, which confirms the chronic inflammatory substrate of psoriasis and the predisposition of patients to metabolic and cardiovascular comorbidities $(18,43)$. Previous studies have found low levels of adiponectin, which has anti-inflammatory, insulin-sensitizing and anti-atherogenic properties, in patients with psoriasis, while other studies have suggested that the production of IL-17 by T cells, which 
serves a role in the pathogenesis of psoriasis, appears to be suppressed by adiponectin, which may indicate an approach to the prevention and even reversibility of metabolic syndrome via administration of low doses of IL-17 (42-45).

\section{Management of metabolic comorbidities in psoriasis}

In the presence of the aforementioned comorbidities, dermatologists may help improve the quality of life of patients with psoriasis through early identification of associated comorbidities, implementation of preventive measures, recommendation of appropriate therapies and close follow-up. In patients with high BMI (overweight or obese) the dermatologist may recommend a healthier lifestyle and weight loss, which would improve both the PASI and the patients' quality of life $(7,9,20,46)$. If standard measures are not effective, surgery, such as gastric bypass, may be considered, as it has been reported to reduce the risk of developing psoriasis and improve the prognosis in patients who already have psoriasis $(9,46-48)$.

As chronic hyperglycemia causes long-term organ dysfunction and several diabetes-related complications are preventable, any pathological glucose level found in patients with psoriasis should alert dermatologists $(9,49)$. Biguanide-type hypoglycemic medication (metformin) is currently the first line of treatment in type 2 diabetes for normalizing blood sugar levels, and it has been reported that good control of psoriasis could be supported by the long-term use of metformin (22). Screening is crucial, given the increased risk of the association between diabetes mellitus and psoriasis. Therefore, early identification of diabetes-specific changes may prove to be an important pillar in treating patients with psoriasis and maintaining their quality of life.

It has been demonstrated that NAFLD is highly associated with psoriasis; therefore, dermatologists should be aware of both the high prevalence of NAFLD in patients with psoriasis, as well as the possible presence of steatohepatitis (which carries the risk of progressing to liver fibrosis, cirrhosis and related complications), which occur predominantly in patients with severe forms of psoriasis or metabolic syndrome $(31,46)$. Since the pathogenic links between psoriasis and NAFLD are chronic inflammation and peripheral insulin resistance, dermatologists should always explore the presence of components of the metabolic syndrome. An abnormal level of liver enzymes, attesting to liver damage, is not always present; thus, ultrasound imaging is recommended even when the level of liver transaminases is normal, as normal findings on initial evaluation do not rule out the development of NAFLD in the following years (46). When NAFLD is confirmed, the dermatologist must inform the patient on the associated risks, recommend dietary measures and adjust the doses of drugs or even change the treatment, avoiding potentially hepatotoxic drugs if transaminase levels are elevated $(46,50)$.

As regards dyslipidemia in psoriasis, the association of other risk factors (such as obesity, hypertension, diabetes mellitus and smoking) may lead to a significant increase in atherosclerosis, cardiovascular disease and stroke risk; therefore, dermatologists must carefully and constantly monitor the serum lipid profile in all patients with psoriasis $(32,33,51)$. The recommended measures are as follows: Dietary restrictions, weight control and pharmacological therapeutic measures.
Statin therapy may be beneficial for patients with psoriasis and dyslipidemia, as statins reduce LDL oxidation and have immunomodulatory activities with beneficial effects on psoriatic skin $(33,52)$. Therapy with TNF- $\alpha$ inhibitors for psoriasis has also been reported to exert a beneficial effect on atherogenic dyslipidemia $(33,34)$.

Since the risk factors for metabolic syndrome and its components are closely associated with psoriasis, dermatologists must inform their patients on this association and ensure that the patients are properly screened for each component, as each component of the syndrome may be targeted by specific treatment. General screening measures for metabolic syndrome include height, weight, BMI, abdominal circumference, fasting blood sugar, HbA1c, cholesterol and triglyceride levels, blood pressure and heart rate. The role of the dermatologist is to recommend a healthy lifestyle (balanced diet, regular exercise, smoking cessation and mental well-being), advise and guide patients with psoriasis to screening and prescribe specialized therapy for each comorbidity $(9,23)$.

\section{Conclusions}

As reported in the present review, psoriasis is a multisystemic inflammatory disease that is associated with multiple comorbidities, the identification of which is a crucial step in the holistic approach of the patient. The aim of the present review was to demonstrate that the current literature increasingly supports the association of metabolic comorbidities with psoriasis and their overlap with severe forms of psoriasis, with a markedly higher prevalence compared with the general population. This association can be highlighted by the common pathophysiological substrate, as supported by previous studies $(2,18,23,43)$. Metabolic comorbidities increase the difficulty of managing patients with psoriasis, and they are associated with an increased risk of cardiovascular events, which highlights the need for these to be identified, prevented and treated to the greatest possible extent. Dermatologists are usually the first medical contact of patients with psoriasis, and they must ensure early identification and management of these comorbidities.

Furthermore, as shown by numerous studies, proper management of metabolic comorbidities also contributes significantly to the treatment of the underlying pathology in psoriasis $(43,46,50)$. These findings emphasize the important role of the dermatologist in the management of patients with psoriasis, as this disease is not limited to the skin, but affects the whole organism.

\section{Acknowledgements}

Not applicable.

\section{Funding}

Publishing funds were supported by the Association of Dermatologists from Moldova.

\section{Availability of data and materials}

All information provided in this review is documented by relevant references. 


\section{Authors' contributions}

DEB, DCB and ACN contributed to the study design, participated in the entire review process and prepared the manuscript. $\mathrm{AD}, \mathrm{MC}, \mathrm{CIB}$ and AES contributed to collecting the relevant literature, data analysis and critical interpretation. RAP, EAP, MPT and AIA conceived the present review and modified the manuscript. All authors have read and approved the final version of the manuscript for publication.

\section{Ethics approval and consent to participate}

Not applicable.

\section{Patient consent for publication}

Not applicable.

\section{Competing interests}

All the authors declare that they have no competing interests.

\section{Authors' information}

Author CIB is a medical student at 'Grigore T. Popa' University of Medicine and Pharmacy Iasi, Romania

\section{References}

1. Puig L: Cardiometabolic comorbidities in psoriasis and psoriatic arthritis. Int J Mol Sci 19: 58, 2017.

2. Jensen P and Skov L: Psoriasis and obesity. Dermatology 232: 633-639, 2016

3. Batani A, Brănișteanu DE, Ilie MA, Boda D, Ianosi S, Ianosi G and Caruntu C: Assessment of dermal papillary and microvascular parameters in psoriasis vulgaris using in vivo reflectance confocal microscopy. Exp Ther Med 15: 1241-1246, 2018.

4. Maranduca MA, Hurjui LL, Branisteanu DC, Serban DN, Branisteanu DE, Dima N and Serban IL: Skin-a vast organ with immunological function (review). Exp Ther Med 20: 18-23, 2020.

5. Grechin C, Solovăstru LG, Vâță D, Pătrașcu AI, Grăjdeanu AI and Andrese-Porumb E: Inflammatory marker alteration in response to systemic therapies in psoriasis. Exp Ther Med 20: 42-46, 2020

6. Gottlieb AB and Dann F: Comorbidities in patients with psoriasis. Am J Med 122: 1150.e1-1150.e9, 2009.

7. Porumb-Andrese E, Vâță D, Postolică R, Stătescu L, Stătescu C, Grăjdeanu AI, Pătrașcu AI, Popescu IA and Solovastru LG: Association between personality type, affective distress profile and quality of life in patients with psoriasis vs. patients with cardiovascular disease. Exp Ther Med 18: 4967-4973, 2019.

8. Alsufyani MA, Golant AK and Lebwohl M: Psoriasis and the metabolic syndrome. Dermatol Ther 23: 137-143, 2010.

9. Elmets CA, Leonardi CL, Davis DM, Gelfand JM, Lichten J, Mehta NN, Armstrong AW, Connor C, Cordoro KM, Elewski BE, et al: Joint AAD-NPF guidelines of care for the management and treatment of psoriasis with awareness and attention to comorbidities. J Am Acad Dermatol 80: 1073-1113, 2019.

10. Sterry W, Strober BE and Menter A: Obesity in psoriasis: The metabolic, clinical and therapeutic implications. Report of an interdisciplinary conference and review. Br J Dermatol 157: 649-655, 2007.

11. Gisondi P, Bellinato F, Girolomoni G and Albanesi C: Pathogenesis of chronic plaque psoriasis and its intersection with cardio-metabolic comorbidities. Front. Pharmacol 11: 117, 2020.

12. Puig L, Kirby B, Mallbris L and Strohal R: Psoriasis beyond the skin: A review of the literature on cardiometabolic and psychological co-morbidities of psoriasis. Eur J Dermatol 24: 305-311, 2014.
13. Lønnberg AS, Skov L, Skytthe A, Kyvik KO, Pedersen OB and Thomsen SF: Association of psoriasis with the risk for type 2 diabetes mellitus and obesity. JAMA Dermatol 152: 761-767, 2016.

14. Armstrong AW, Harskamp CT and Armstrong EJ: The association between psoriasis and obesity: A systematic review and meta-analysis of observational studies. Nutr Diabetes 2: e54, 2012.

15. Ghiasi M, Nouri M, Abbasi A, Hatami P, Abbasi MA and Nourijelyani K: Psoriasis and increased prevalence of hypertension and diabetes mellitus. Indian J Dermatol 56: 533-536, 2011.

16. Shapiro J, Cohen AD, David M, Hodak E, Chodik G, Viner A, Kremer E and Heymann A: The association between psoriasis, diabetes mellitus, and atherosclerosis in Israel: A case control study. J Am Acad Dermatol 56: 629-634, 2007.

17. Granata M, Skarmoutsou E, Trovato C, Rossi GA, Mazzarino MC and D'Amico F: Obesity, type 1 diabetes, and psoriasis: An autoimmune triple flip. Pathobiology 84" 71-79, 2016.

18. Cohen AD, Sherf M, Vidavsky L, Vardy DA, Shapiro J and Meyerovitch J: Association between psoriasis and the metabolic syndrome. A cross-sectional study. Dermatology 216: 152-155, 2008.

19. Bartosińska J, Przepiórka-Kosińska J, Sarecka-Hujar B, Raczkiewicz D, Kowal M, Chyl-Surdacka K, Bartosiński J, Kosiński J, Krasowska D and Chodorowska G: Osteopontin serum concentration and metabolic syndrome in male psoriatic patients. J Clin Med 10: 755, 2021.

20. Dai YX, Shen YJ, Chou YJ, Chang YT, Chen TJ, Li CP and Wu CY: Obesity, but not metabolic diseases, is associated with risk of psoriasis: A population-based cohort study in taiwan. Dermatology 236: 521-528, 2020.

21. Owczarczyk-Saczonek A, Purzycka-Bohdan D, Nedoszytko B, Reich A,Szczerkowska-DoboszA,BartosiñskaJ,Batycka-Baran A, Czajkowski R, Dobrucki IT, Dobrucki LW, et al: Pathogenesis of psoriasis in the 'omic' era. Part III. Metabolic disorders, metabolomics, nutrigenomics in psoriasis in psoriasis. Postepy Dermatol Alergol 37: 452-467, 2020.

22. Yamazaki F: Psoriasis: Comorbidities. J Dermatol 48: 732-740, 2021.

23. Brazzelli V, Maffioli P, Bolcato V, Ciolfi C, D'Angelo A, Tinelli C and Derosa G: Psoriasis and diabetes, a dangerous association: Evaluation of insulin resistance, lipid abnormalities, and cardiovascular risk biomarkers. Front Med (Lausanne) 8: 605691, 2021.

24. Brănişteanu DE, Voicu CM, Creţu A, Dimitriu A, Luca MC and Sălăvăstru CM: Adverse reactions of biological therapy for psoriasis. Rev Med Chir Soc Med Nat Iasi 119: 38-44, 2015.

25. Committee of the Japan Diabetes Society on the Diagnostic Criteria of Diabetes Mellitus, Seino Y, Nanjo K, Tajima N, Kadowaki T, Kashiwagi A, Araki E, Ito C, Inagaki N, Iwamoto Y, et al: Report of the committee on the classification and diagnostic criteria of diabetes mellitus. J Diabetes Investig 1: 212-228, 2010.

26. Radtke MA, Mrowietz U, Feuerhahn J, Härter M, von Kiedrowski R, Nast A, Reich K, Strömer K, Wohlrab J and Augustin M: Early detection of comorbidity in psoriasis: recommendations of the national conference on healthcare in psoriasis. J Dtsch Dermatol Ges 13: 674-690, 2015 (In English, German).

27. Armstrong AW, Harskamp CT and Armstrong EJ: Psoriasis and the risk of diabetes mellitus. JAMA Dermatol 149: 84-91, 2013.

28. Li W, Han J, Hu FB, Curhan GC and Qureshi AA: Psoriasis and risk of type 2 diabetes among women and men in the United States: A population-based cohort study. J Invest Dermatol 132: 291-298, 2012.

29. Tula E, Ergun T, Seckin D, Ozgen Z and Avsar E: Psoriasis and the liver: Problems, causes and course. Australas J Dermatol 58: 194-199, 2017.

30. Gisondi P, Targher G, Zoppini G and Girolomoni G: Non-alcoholic fatty liver disease in patients with chronic plaque psoriasis. J Hepatol 51: 758-764, 2009.

31. Van der Voort EA, Koehler EM, Dowlatshahi EA, Hofman A, Stricker BH, Janssen HLA, Schouten JNL and Nijsten T: Psoriasis is independently associated with nonalcoholic fatty liver disease in patients 55 years old or older: Results from a population-based study. J Am Acad Dermatol 70: 517-524, 2014.

32. Gisondi P, Ferrazzi A and Girolomoni G: Metabolic comorbidities and psoriasis. Acta Dermatovenerol Croat 18: 297-304, 2010.

33. Ghafoor R, Rashid A and Anwar MI: Dyslipidemia and psoriasis: A case control study. J Coll Physicians Surg Pak 25: 324-327, 2015. 
34. Mocanu.M, Toader MP, Rezus E and Taranu T: Aspects concerning patient adherence to anti-TNFa therapy in psoriasis: A decade of clinical experience. Exp Ther Med 18: 4987-4992, 2019.

35. Grundy SM, Brewer HB Jr, Cleeman JI, Smith SC Jr, Lenfant C, American Heart Association, National Heart, Lung, and Blood Institute: Definition of metabolic syndrome: Report of the national heart, lung, and blood institute/American heart association conference on scientific issues related to definition. Circulation 109: 433-438, 2004.

36. Elgendy A, Alshawadfy E, Altaweel A and Elsaidi A: Cardiovascular and metabolic comorbidities of psoriasis. Dermatol Case Rep 1: 106, 2016.

37. Gisondi P, Tessari G, Conti A, Piaserico S, Schianchi S, Peserico A, Giannetti A and Girolomoni G: Prevalence of metabolic syndrome in patients with psoriasis: A hospital-based case-control study. Br J Dermatol 157: 68-73, 2007.

38. Singh S, Young P and Armstrong AW: Relationship between psoriasis and metabolic syndrome: A systematic review. G Ital Dermatol Venereol 151: 663-677, 2016.

39. Goldminz AM, Buzney CD, Kim N, Au SC, Levine DE, Wang AC, Volf EM, Yaniv SS, Kerensky TA, Bhandarkar M, et al: Prevalence of the metabolic syndrome in children with psoriatic disease. Pediatr Dermatol 30: 700-705, 2013.

40. Gisondi P, Fostini AC, Fossà I, Girolomoni G and Targher G: Psoriasis and the metabolic syndrome. Clin Dermatol 36: 21-28, 2018.

41. Wolk K, Mallbris L, Larsson P, Rosenblad A, Vingård E and Ståhle M: Excessive body weight and smoking associates with a high risk of onset of plaque psoriasis. Acta Derm Venereol 89: 492-497, 2009.

42. Von Stebut E, Boehncke WH, Ghoreschi K, Gori T, Kaya Z, Thaci D and Schäffler A: IL-17A in psoriasis and beyond: Cardiovascular and metabolic implications. Front Immunol 10: 3096, 2020

43. Gottlieb AB, Chao C and Dann F: Psoriasis comorbidities. J Dermatolog Treat 19: 5-21, 2008
44. Lynde CW, Poulin Y, Vender R, Bourcier M and Khalil S: Interleukin 17A: Toward a new understanding of psoriasis pathogenesis. J Am Acad Dermatol 71: 141-150, 2014

45. Wolk K and Sabat R: Adipokines in psoriasis: An important link between skin inflammation and metabolic alterations. Rey Endocr Metab Disord 17: 305-317, 2016.

46. Dauden E, Blasco AJ, Bonanad C, Botella R, Carrascosa JM, González-Parra E, Jodar E, Joven B, Lázaro P, Olveira A, et al: Position statement for the management of comorbidities in psoriasis. J Eur Acad Dermatol Venereol 32: 2058-2073, 2018.

47. Takeshita J, Grewal S, Langan SM, Mehta NN, Ogdie A, Van Voorhees AS and Gelfand JM: Psoriasis and comorbid diseases. Epidemiology. J Am Acad Dermatol 76: 377-390, 2017.

48. Langan SM, Seminara NM, Shin DB, Troxel AB, Kimmel SE, Mehta NN, Margolis DJ and Gelfand JM: Prevalence of metabolic syndrome in patients with psoriasis: A population-based study in the United Kingdom. J Invest Dermatol 132: 556-562, 2012.

49. Christophers E: Comorbidities in psoriasis. Clin Dermatol 25: 529-534, 2007

50. Korman NJ: Management of psoriasis as a systemic disease: What is the evidence? Br J Dermatol 182: 840-848, 2020.

51. Wohlrab J, Fiedler G, Gerdes S, Nast A, Philipp S, Radtke MA, Thaçi D, Koenig W, Pfeiffer AF, Härter M and Schön MP: Recommendations for detection of individual risk for comorbidities in patients with psoriasis. Arch Dermatol Res 305: 91-98, 2013.

52. Ryan C and Kirby B: Psoriasis is a systemic disease with multiple cardiovascular and metabolic comorbidities. Dermatol Clin 33: $41-55,2015$

cc) (i) $\odot$ This work is licensed under a Creative Commons CY NC ND Attribution-NonCommercial-NoDerivatives 4.0 International (CC BY-NC-ND 4.0) License. 